\title{
A Potential Method for Identifying Minerals in Comet Samples Using Raman Spectroscopy with a Laser Scanning Confocal Microscope
}

\author{
Amanda J. White and Denton S. Ebel
}

Dept. of Earth and Planetary Sciences, American Museum of Natural History, New York, NY 10024

The NASA Stardust mission to comet Wild2 returned to Earth in 2006 with an aerogel collector containing trapped cometary particles. The cometary particles were captured at a relative velocity of 6.1 $\mathrm{km} / \mathrm{s}$, creating three-dimensional (3D) tracks containing compressed aerogel, melted aerogel, fragmented cometary material, and void space [1]. Each track represents a unique hypervelocity impact event that stores information on the nature of the impact, including the state of the original impactor, in the 3D morphology and material distribution along the track.

Using a Zeiss LSM 710, a laser scanning confocal microscope (LSCM), located at the Microscopy and Imaging Facility at the American Museum of Natural History, we acquire high resolution 3D image stacks of Stardust tracks in order to characterize track morphology [2,3,4]. Complementary chemical data is acquired using the GSECARS X-ray microprobe on beamline 13-IDE at the Advanced Photon Source (APS) of Argonne National Laboratory. As an in-house, bench top instrument, LSCM quickly produces high resolution images of in situ particles larger than $100 \mathrm{~nm}$ and compressed aerogel along an entire track without disturbing or destroying the sample [2,3,4]. Obtaining X-ray data is not simple, because time at APS is both limited and highly competitive. For this reason, we have investigated the utility of grafting a Raman spectrometer onto our existing LSCM system. The LSM 710 is equipped with a side port that allows for the addition of an additional detector. By first passing light through the existing optics of the LSM 710 we anticipate being able to take advantage of the instrument's high spatial resolution and spectroscopic capabilities to obtain compositional data on cometary particles at very high spatial resolution.

Raman spectroscopy is a technique that can identify minerals and amorphous materials by spectral measurement of the inelastic scattering of photons and the wavelength shifts associated with scattered photons in a reflection mode. The signatures of inelastic scattering of photons from a monochromatic laser can be used as a molecular fingerprint to identify the composition of target grains in a similar fashion to the way fluorescence due to molecular excitations from high energy X-rays is used to determine composition in synchrotron X-ray fluorescence. Raman spectroscopy requires minimal sample preparation, making this technique an ideal method for working with delicate Stardust cometary keystones in a nondestructive manner.

Successful application of Raman spectroscopy to aerogel keystones has previously been demonstrated by other groups using aerogel samples that were shot with known mineral and meteoritic grains by a light gas gun [5,6]. Sizes of shot particles ranged from 50-100 microns [5] and $200-300$ microns [6]. Of the 13 mineral types they tested, recognizable Raman spectra were obtained for all of the materials that were successfully fired into aerogel [5]. Furthermore, compositions of the meteoritic samples could be identified readily using Raman spectra freely available in the U. Arizona RRUFF database [7]. This indicates that the presence of aerogel does not alter the spectrum of a captured grain to the point that it cannot be recognized using a standard database. The biggest difference between our method and the standard Raman setup used in previous experiments is the addition of the superior spatial resolution 
provided by the LSM 710. Previous studies mapped samples with a spatial resolution of $5-10$ microns [6]. While [5,6] had difficulty finding even the largest grains using a light microscope [8], we do not anticipate that this will be a problem with our intended setup. By first scanning allocated Stardust tracks using the LSM 710's QUASAR detector in reflectance mode, we can pinpoint particle locations before switching to the Raman spectrometer. This switch can be done without moving the stage and without losing focus on the grain of interest.

We anticipate that the unprecedented and innovative coupling of Raman spectroscopy with high resolution laser confocal microscopy, if funded, will allow accurate and rapid first-order characterization of at least the metallic or silicate nature of grains in Stardust sample keystones. Further work may allow distinction between pyroxenes and olivines.

\section{References:}

[1] D Brownlee et al., Science 314 (2006), p. 1711 - 1716.

[2] M Greenberg and DS Ebel, Meteoritics and Planetary Science 47 (2012), p. $634-648$.

[3] M Greenberg and DS Ebel, Geosphere 6 (2010), p. 515 - 523.

[4] DS Ebel et al., Meteoritics and Planetary Science 44 (2009), p. $1454-1463$.

[5] MJ Burchell et al., Meteoritics and Planetary Science 41 (2006), p. 217 - 232.

[6] MJ Burchell, JA Creighton, and AT Kearsley, Journal of Raman Spectroscopy 35 (2004), p.249-253.

[7] RT Downs, 19th General Meeting of the International Mineralogical Association (2006) O03-13.

[8] MJ Burchell, personal communication.

[9] The authors acknowledge funding from NASA LARS grant NNX12AF28G (DSE). The LSM 710 was acquired by AMNH with partial support from NASA LARS grant NNXIOAH06G (DSE). 